\title{
Electronic and atomic structures of the $\mathrm{Si}-\mathrm{C}-\mathrm{N}$ thin film by $\mathrm{x}$-ray-absorption spectroscopy and theoretical calculations
}

\author{
Y. K. Chang, H. H. Hsieh, and W. F. Pong* \\ Department of Physics, Tamkang University, Tamsui, Taiwan 251, Republic of China \\ M.-H. Tsai \\ Department of Physics, National Sun Yat-Sen University, Kaohsiung, Taiwan 804, Republic of China \\ K. H. Lee \\ Department of Physics, Tamkang University, Tamsui, Taiwan 251, Republic of China \\ T. E. Dann \\ Department of Physics, Tamkang University, Tamsui, Taiwan 251, Republic of China \\ and Synchrotron Radiation Research Center, Hsinchu, Taiwan 300, Republic of China \\ F. Z. Chien and P. K. Tseng \\ Department of Physics, Tamkang University, Tamsui, Taiwan 251, Republic of China \\ K. L. Tsang \\ Synchrotron Radiation Research Center, Hsinchu, Taiwan 300, Republic of China \\ W. K. Su \\ Department of Applied Physics, Chung-Cheng Institute of Technology, Ta-Hsi, Taiwan 335, Republic of China \\ L. C. Chen and S. L. Wei \\ Center for Condensed Matter Sciences, National Taiwan University, Taipei, Taiwan 107, Republic of China \\ K. H. Chen and D. M. Bhusari \\ Institute of Atomic and Molecular Sciences, Academia Sinica, Taipei, Taiwan 107, Republic of China \\ Y. F. Chen \\ Department of Physics, National Taiwan University, Taipei, Taiwan 107, Republic of China
}

(Received 24 October 1997; revised manuscript received 13 April 1998)

\begin{abstract}
This study measures the x-ray-absorption spectra of a crystalline $(c)$-Si-C-N thin film at the $\mathrm{C}$ and $\mathrm{Si} K$ edge using the sample drain current mode and at the $\mathrm{N} K$ edge using the fluorescence mode. A resonance peak resembling the $\mathrm{C} 1 s$ core exciton in the chemical-vapor-deposition-diamond/Si is observed. In addition, a broad feature is found in the energy range between $\sim 290$ and $305 \mathrm{eV}$, which can be assigned to the antibonding C $2 p$-Si $3 s p$ hybridized states and the $\mathrm{C} 2 p-\mathrm{N} 2 s p$ hybridized states as well. The fact that the resonance peak is located $\sim 1.5 \mathrm{eV}$ below the $\mathrm{C} 1 s$ ionization energy suggests that the Frenkel-type exciton model can appropriately describe the core exciton of carbon atoms in $c$-Si-C-N. Closely examining the $\mathrm{N} K$ edge near edge absorption spectra reveals similar features in both $c-\mathrm{Si}-\mathrm{C}-\mathrm{N}$ and $\alpha-\mathrm{Si}_{3} \mathrm{~N}_{4}$, indicating that nitrogen atoms generally have a similar local environment in these two materials. Moreover, results obtained from Si $K$-edge absorption spectra of $c$-Si-C-N demonstrate a proportional combination of local $\mathrm{Si}-\mathrm{N}$ and $\mathrm{Si}-\mathrm{C}$ bonds associated with the local tetrahedral $\mathrm{C}-\mathrm{Si}-\mathrm{N}_{3}$ arrangement as well as the long-range ordered atomic structure around $\mathrm{Si}$ atoms. Theoretical calculations using the first-principles pseudofunction method are also presented and compared with experimental data. [S0163-1829(98)08037-0]
\end{abstract}

\section{INTRODUCTION}

Silicon carbide (Si-C) and silicon nitride (Si-N) are highly promising for a wide range of applications in mechanics, optics, and electronics. ${ }^{1}$ Carbon nitride $(\mathrm{C}-\mathrm{N})$, another binary compound, has also received extensive attention recently owing to its potential applications in new hard materials. ${ }^{2}$ It is expected that the ternary Si-C-N system may be similarly important. ${ }^{3}$ Gheorghiu et al. ${ }^{4}$ and Ténégal et al. ${ }^{5}$ recently performed Si $K$-edge extended x-ray-absorption fine struc- ture (EXAFS) studies of nanometric Si-C-N powders. However, they obtained different atomic structures. Gheorghiu et al. found a local $\mathrm{C}-\mathrm{Si}-\mathrm{N}_{3}$ arrangement around $\mathrm{Si}$ atoms, while Ténégal et al. concluded that the $\mathrm{Si}$ atoms are randomly linked together through the C-N network. Another investigation found the existence of mixed coordinations around silicon atoms, as evidenced by an extended energy loss fine structure analysis at the $\mathrm{Si} K$ edge of the Si-C-N thin film. ${ }^{6}$ Meanwhile, an x-ray-spectroscopy study of a local structure for nanometric Si-C-N powders indicated that the 
local environment of silicon atoms are strongly dependent on the N/C concentration ratio. ${ }^{7}$ So far the local atomic structure in Si-C-N are still not well understood and the $\mathrm{C}$ and $\mathrm{N}$ $K$-edge $\mathrm{x}$-ray-absorption spectra as well as the electronic structure have not been studied, yet. In this study, we analyzed the electronic and atomic structures of the crystalline (c)-Si-C-N thin film obtained by combining $\mathrm{C}, \mathrm{N}$, and $\mathrm{Si}$ $K$-edge $\mathrm{x}$-ray-absorption measurements and first-principles electronic structure calculation using the pseudofunction method. Results presented herein provide further insight into the local electronic and atomic structures in the ternary $\mathrm{Si}$ C-N system.

\section{EXPERIMENT}

The C, N, and Si $K$-edge X-ray-absorption spectra of the $c$-Si-C-N thin film were measured with that of chemicalvapor-deposition (CVD)-grown diamond on $\mathrm{Si}$ substrate, $\alpha-\mathrm{Si}_{3} \mathrm{~N}_{4}$ powders, and thin film $\beta-\mathrm{SiC}$ and $c-\mathrm{Si}(100)$ given as references. The measurement was performed using the highenergy spherical grating monochromator (HSGM) and InSb(111) double crystal monochromator (DCM) beamlines with an electron-beam energy of $1.5 \mathrm{GeV}$ and a maximum stored current of $200 \mathrm{~mA}$ at the Synchrotron Radiation Research Center (SRRC), Hsinchu, Taiwan. The spectra of the $\mathrm{C}$ and $\mathrm{Si} K$ edge were measured using the sample drain current mode and the fluorescence measurements for the $\mathrm{N} K$ edge spectra were taken with a high-sensitivity sevenelement $\mathrm{Ge}$ detector at room temperature. Photon energies for the $\mathrm{C}$ and $\mathrm{N} K$-edge $\mathrm{x}$-ray-absorption near edge structure (XANES) spectra were calibrated using the bulk diamond ${ }^{8}$ and the well known $\mathrm{CaF}_{2}$ thin film and the $\mathrm{Si} K$-edge XANES spectra were calibrated using the $c$-Si(100) film. ${ }^{9}$ The typical resolution of the spectra was $0.2 \mathrm{eV}$ for HSGM and $0.7 \mathrm{eV}$ for DCM beamlines. The $c$-Si-C-N sample was grown by microwave plasma-enhanced chemical vapor deposition process on $c-\mathrm{Si}(100)$. Details of the preparation procedure for similar compounds have been described elsewhere. ${ }^{10}$ X-ray-diffraction (XRD) spectra of $c$-Si-C-N exhibited many sharp lines, of which the most distinct ones mimic those of $\alpha-\mathrm{Si}_{3} \mathrm{~N}_{4}$ as shown in Fig. 1, though deviations of relative intensities and peak positions from those of $\alpha-\mathrm{Si}_{3} \mathrm{~N}_{4}$ can be seen. These XRD data clearly showed that there was no significant contribution from the $\beta$-SiC phase in $c$-Si-C-N because they did not have the three pronounced diffraction peaks located at $2 \theta \cong 35.8,60.2$, and $72.0^{\circ}$ generally observed in the $\beta$-SiC. ${ }^{11} \mathrm{XRD}$ analysis yielded that the polycrystalline $c$-Si-C-N was dominated by a structure with similar crystal symmetries of the $\alpha-\mathrm{Si}_{3} \mathrm{~N}_{4}$ structure characterized by a hexagonal structure with a corner-linked $\mathrm{SiN}_{4}$ tetrahedra. ${ }^{12}$ The scanning electron microscopy micrograph of $c$-Si-C-N also reveals a randomly oriented image, in which large grains are formed with sizes of the order of 0.5 $\mu \mathrm{m}$ on the Si substrate. The O $K$-edge XANES and Si $K$ edge EXAFS measurements as presented in the following section shows negligible oxidation of the $c-\mathrm{Si}-\mathrm{C}-\mathrm{N}$ film.

\section{THEORY}

A first-principles pseudofunction (PSF) method ${ }^{13}$ was employed to calculate the projected density of states (DOS) of

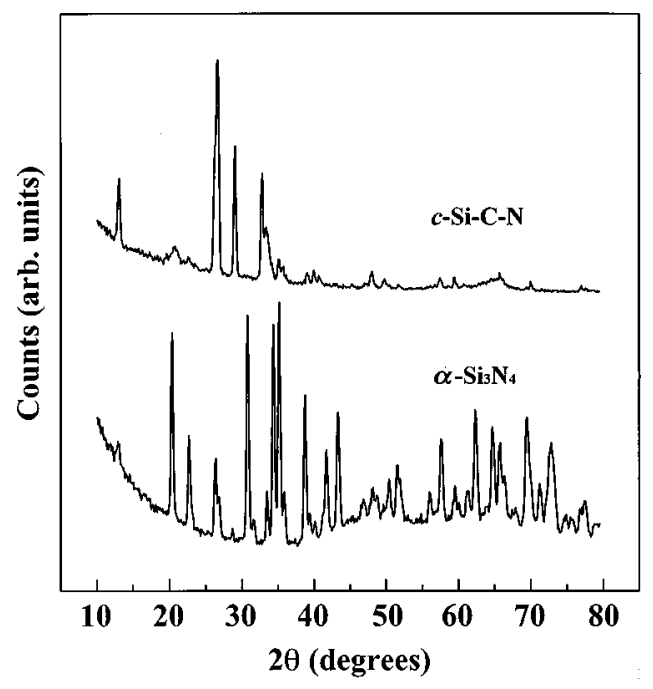

FIG. 1. Representative x-ray-diffraction spectra of polycrystalline $c$-Si-C-N and $\alpha-\mathrm{Si}_{3} \mathrm{~N}_{4}$.

the $c$-Si-C-N compound. In this method, the potential was divided into spherical potentials within the muffin-tin spheres and a plane-wave expanded potential that extends throughout the crystal, which includes the interstitial potential and the nonspherical part of potentials within muffin-tin spheres. PSF's are smooth mathematical functions constructed by continuously and differentially extending the muffin-tin-orbitals tails into muffin-tin spheres. They are simply devices to calculate the interstitial and nonspherical parts of matrix elements efficiently ${ }^{13}$ through plane waves using the fast-Fourier technique. In this study, we expanded PSF's in $13125(25 \times 25 \times 21)$ plane waves. The interstitial and nonspherical parts of the charge density and potential were expanded in $98441(49 \times 49 \times 41)$ plane waves. Six special $\left(\mathbf{k}_{x}, \mathbf{k}_{y}\right)$ points of Cunningham for the two-dimensional hexagonal lattice with $\mathbf{k}_{z}=\frac{1}{4}(2 \pi / c)$ were used to obtain the self-consistent charge density and potential. ${ }^{14}$ To obtain the partial DOS, we have sampled $\mathbf{k}_{z}=\frac{1}{16}, \frac{3}{16}, \frac{5}{16}$, and $\frac{7}{16}(2 \pi / c)$. For each $\mathbf{k}_{z}$, we chose the six special $\left(\mathbf{k}_{x}, \mathbf{k}_{y}\right)$ points of Cunningham for the two-dimensional hexagonal Bravais lattice. Totally, we have sampled twenty-four special $\mathbf{k}$ points for obtaining the partial DOS. An x-ray-diffraction measurement revealed that $c-\mathrm{Si}-\mathrm{C}-\mathrm{N}$ has a $\alpha-\mathrm{Si}_{3} \mathrm{~N}_{4}$ structure with lattice constants $a=13.055$ a.u. and $c=9.943$ a.u. The unit-cell parameters and atomic positions were determined from the X-ray-crystallograph table. ${ }^{15}$ Muffin-tin radii of 2.0, 1.0, and 1.2 a.u. were chosen for $\mathrm{Si}, \mathrm{C}$, and $\mathrm{N}$, respectively, which are roughly proportional to their covalent radii ${ }^{16}$ with the constraint that all muffin-tin spheres do not overlap.

\section{RESULTS AND DISCUSSION}

Figure 2(a) plots the $\mathrm{C} K$-edge XANES spectra of $\beta$-SiC, $c$-Si-C-N, and CVD-grown diamond on Si substrate (abbreviated hereafter as diamond/Si) for comparison. After preedge background subtraction, the spectra were normalized using the incident beam intensity $I_{0}$ and then scaled to the maximum peak heights. For x-ray energies in the XANES region, the excited photoelectron undergoes a transition from a core level to an unoccupied state; the final state is determined in relation to the initial state by the dipole selection 

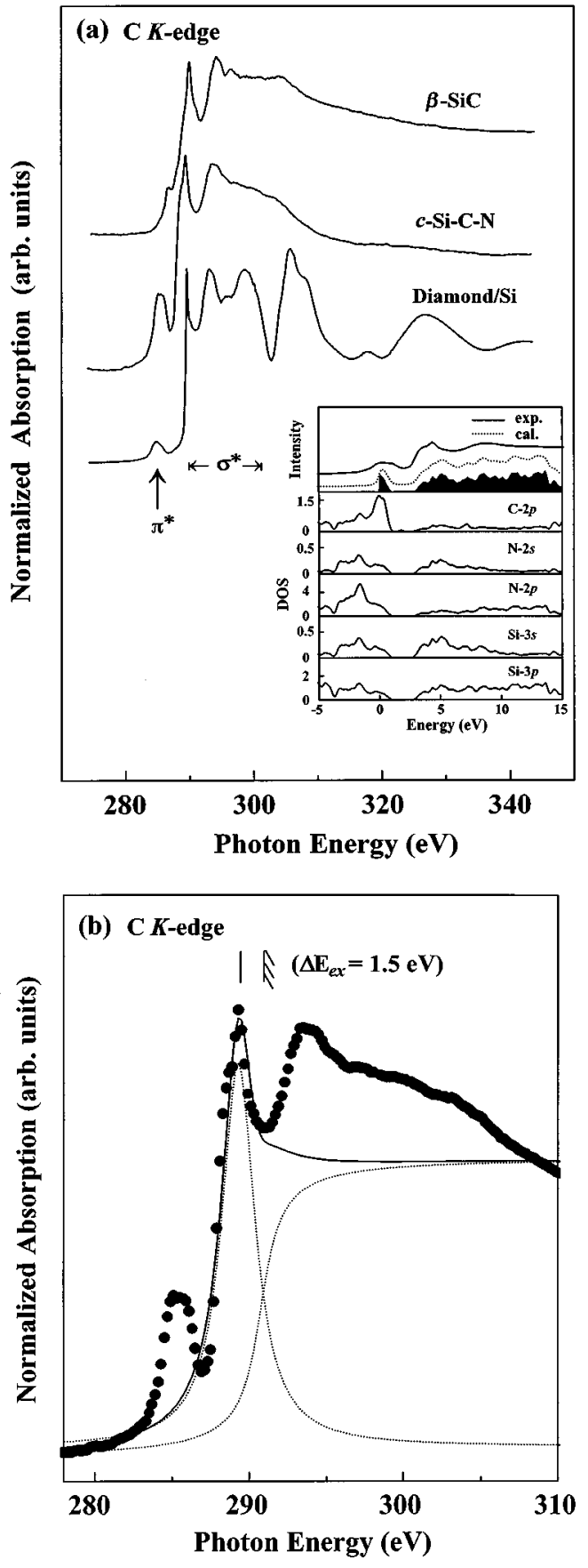

FIG. 2. (a) Normalized C $K$-edge absorption spectra of $\beta$-Si-C, $c$-Si-C-N, and diamond/Si. The inset is a comparison of the $\mathrm{C} K$ edge XANES spectra with calculated carbon $p$-projected density of states (DOS) of $c$-Si-C-N. The darkened area shows the theoretical carbon $p$-projected DOS above the Fermi level, which is defined as the zero energy. The upper solid curve is the $\mathrm{C} K$-edge absorption spectra at and above the threshold and the dashed curve in the middle is the theoretical carbon $p$-projected DOS broadened with a Lorentzian and convoluted with a Gaussian function. The spectra have been aligned at the position of the first main peak, and the intensity units have been normalized arbitrarily. (b) The part of Fig. 1 (a) in the vicinity of the $\mathrm{C} 1 s$ core exciton resonance about $4 \mathrm{eV}$ above the Fermi level. The solid lines were calculated by a summation of the Lorentzian peak and the arctangent function shown by the dashed lines. The experimental data are shown by the filled circles. rule. It is well known that carbon atoms in diamond form strong tetrahedrally coordinated $s p^{3}$ hybrid bonds. The spectra of diamond/Si clearly display a sharp feature, which closely resembles that reported in earlier works. ${ }^{8,17}$ The XANES energy range for diamond generally can be divided into two regions characterized by their specific features, namely, (a) at $\sim 289.2 \mathrm{eV}$ is the $\mathrm{C} 1 s$ core exciton resonance and (b) between 290 and $302 \mathrm{eV}$ is a relatively broad band of $\sigma^{*}$ states of $s p^{3}$ bonded carbon atoms. ${ }^{8}$ The relatively small peak at $\sim 285 \mathrm{eV}$ obviously appears due to non-diamond-like $s p^{2}$ bonded carbon of $\pi^{*}$ states. ${ }^{18,19}$ In contrast, the carbon atoms in $c$-Si-C-N and $\beta$-SiC have different local environments than those of carbon atoms in diamond/Si. Thus, the $\mathrm{C}$ $K$-edge XANES spectra of $c$-Si-C-N and $\beta$-SiC are somewhat different than that of diamond/Si. According to Fig. 2 (a), the spectra of $c-\mathrm{Si}-\mathrm{C}-\mathrm{N}$ are quite close to that of $\beta$-SiC. Both spectra contain a weak structure at the pre-edge region and display a strong and sharp excitonic peak immediately above the threshold. These spectra have broad features above the edge up to $\sim 305 \mathrm{eV}$. The broader feature in the nearedge region in $\beta$-SiC can be attributed to dipole-allowed transitions of photoelectrons from the $\mathrm{C} 1 s$ states to the unoccupied $2 p$ states. These states have been hybridized significantly with the $\mathrm{Si} 3 s p$ states according to the calculated DOS of $\beta$-SiC. ${ }^{20}$ In the inset of Fig. 2(a), the C $K$-edge XANES spectra are compared with calculated carbon $p$ projected DOS of $c$-Si-C-N. The darkened area shows the theoretical carbon $p$-projected DOS above the Fermi level. The upper solid curve is the $\mathrm{C} K$-edge absorption spectra at the region of XANES. In addition, the dashed curve in the middle represents the theoretical carbon $p$-projected DOS broadened with a Lorentzian of $0.2 \mathrm{eV}$ to simulate the corehole lifetime ${ }^{8}$ and convoluted with a Gaussian function of $0.2 \mathrm{eV}$ to simulate the experimental resolution. According to the inset of Fig. 2(a), the partial density of $\mathrm{N} 2 s p$ and $\mathrm{Si} 3 s p$ states spreads over a wide range with a gap from about 1 up to about $3 \mathrm{eV}$ above the Fermi level. The $\mathrm{C} 1 s$ near-edge feature of $c-\mathrm{Si}-\mathrm{C}-\mathrm{N}$ appears to be due to dipole transitions from the $\mathrm{C} 1 s$ states to the unoccupied $2 p$ states, which have also been hybridized significantly with the $\mathrm{N} 2 s p$ and Si $3 s p$ states. Our theoretical calculations indicate that the N $2 p$ and Si $3 p$ have a larger DOS above the threshold region than that of the $\mathrm{N} 2 s$ and $\mathrm{Si} 3 s$ states. The $\mathrm{C} 1 s$ core exciton resonance of $c$-Si-C-N show a broader feature than that of diamond/Si. We can say that this broader resonance peak can be attributed primarily to the $\mathrm{C} 1 s$ core excitonic state, which also mixed with some contributions from the antibonding $\mathrm{C} 2 p-\mathrm{Si}$ $3 s p$ and $\mathrm{C} 2 p-\mathrm{N} 2 s p$ hybridized states. Furthermore, the preedge $\pi^{*}$-like peak in the $c$-Si-C-N spectra increases in intensity and its position is shifted slightly to the higher energy side. Based on the calculational results, the pre-edge $\pi^{*}$-like peak can be interpreted in terms of the antibonding $\mathrm{C} 2 p-\mathrm{Si}$ $3 s p$ and $\mathrm{C} 2 p-\mathrm{N} 2 s p$ hybridized states. The relatively large $\pi^{*}$-like peak also appears to be due to carbon atoms in $c$-Si$\mathrm{C}-\mathrm{N}$, which has a larger degree of disorder of the $s p^{2}$ bonds similar to diamond/Si. According to an electron-energy-loss spectroscopy study on the structure of nitrogen-doped tetrahedrally bonded amorphous carbon films, ${ }^{21}$ the intensity of $\pi^{*}$-like feature increases with an increasing N/C concentration ratio. This occurrence can be accounted for by the fact that the excess $\mathrm{N}$ doping promotes disordered $s p^{2}$ bonds to incorporate stress relaxation and graphitization. This is fur- 
ther supported by the theoretical calculations for a wide range of stoichiometries in an amorphous carbon nitride system. ${ }^{22}$

C $1 s$ core excitons in diamond have been the subject of controversial debates. Morar et al. have used the hydrogenic effective mass approximation to show that the Wannier-type exciton has a binding energy of $0.19 \mathrm{eV} .{ }^{8}$ Later, Jackson and Pederson suggested that a higher binding energy (of order $1.7 \mathrm{eV}$ ) exists for the Frenkel-type exciton, which is dipole forbidden due to the symmetry selection rule. ${ }^{23}$ Based on x-ray-absorption and emission studies of diamond and graphite and the consideration that the exciton may couple with phonons to give rise to the Jahn-Teller distortion, Ma et al. ${ }^{24}$ proposed a model that incorporates both the Wannier and the Frenkel approaches. To quantitatively address the excitonic binding energy $\Delta E_{\text {ex }}$ of carbon atoms in $c$-Si-C-N, we investigate the $\mathrm{C} 1 s \Delta E_{\text {ex }}$ using the Lorentzian and arctangent functions to describe the excitonic state and the continuous step, ${ }^{25}$ respectively. Figure 2(b) is an enlargement of the part of absorption data and the fitted spectrum in Fig. 2(a) in the vicinity of the excitonic peak. The excitonic peak of $c$-Si$\mathrm{C}-\mathrm{N}$ is located at $\sim 1.5 \mathrm{eV}$ below the $\mathrm{C} 1 s$ ionization energy $(290.8 \mathrm{eV})$ locates at the reflection point of the continuous step. The full width at half maximum (FWHM) of the excitonic state is $2.6 \mathrm{eV}$, which is larger than that of diamond/Si $(\sim 0.5 \mathrm{eV})$. The larger FWHM for $c$-Si-C-N is presumably due to (a) the contribution of antibonding $\mathrm{C} 2 p-\mathrm{Si} 3 s p$ and $\mathrm{C}$ $2 p-\mathrm{N} 2 s p$ hybridized states mentioned earlier and (b) an increase in the degree of disorder of the carbon atoms in $c$-Si$\mathrm{C}-\mathrm{N}$. The excitonic binding energy strongly depends on the local electronic band structures ${ }^{8}$ and bonding environment around the absorbing atoms. ${ }^{26}$ The $\mathrm{C} 1 s \Delta E_{\mathrm{ex}}$ has an order of $1 \mathrm{eV}$, which suggests that the Frenkel-type exciton model can properly describe the core exciton of carbon atoms in $c$-Si-C-N because of the large excitonic binding energy that renders the excitonic level to be well separated from the $\mathrm{C} 1 \mathrm{~s}$ continuous step. According to a more recent study of the band gap of the $c$-Si-C-N film using a piezoreflectance technique, ${ }^{27} c$-Si-C-N is a direct wide-gap semiconductor with a band gap of $\sim 3.81 \mathrm{eV}$ at $300 \mathrm{~K}$. This finding correlates with the Frenkel-type exciton with a strong electroncore-hole interaction that has been found in wide-gap materials. $^{28}$

Figure 3 displays the photon-flux-normalized $\mathrm{N} K$-edge XANES spectra of $c$-Si-C-N and $\alpha-\mathrm{Si}_{3} \mathrm{~N}_{4}$. This figure reveals splitting double-peak features near 403.8 and $406.0 \mathrm{eV}$ in the $\alpha-\mathrm{Si}_{3} \mathrm{~N}_{4}$ spectrum (labeled as two vertical dashed lines). A similar splitting feature is also observed in the $c$ $\mathrm{Si}-\mathrm{C}-\mathrm{N}$ spectrum. Based on the calculated DOS of $\mathrm{N} 2 p$ symmetry, ${ }^{29}$ the white line features in the $\mathrm{N} K$-edge spectrum of $\alpha-\mathrm{Si}_{3} \mathrm{~N}_{4}$ can be attributed to the antibonding N $2 p$-Si $3 s p$ hybridized states. The similarity in the spectra of both $c$-Si$\mathrm{C}-\mathrm{N}$ and $\alpha-\mathrm{Si}_{3} \mathrm{~N}_{4}$ suggests that nitrogen atoms overall have similar local environment in both materials. The inset of Fig. 3 compares the $\mathrm{N} K$-edge XANES spectra with the calculated unoccupied nitrogen $p$-projected DOS of $c$-Si-C-N. The darkened area represents the theoretical nitrogen $p$-projected DOS above the Fermi level. The upper solid curve depicts the $\mathrm{N} K$-edge absorption spectra of white line features. The dashed curve in the middle is the theoretical nitrogen $p$ projected DOS broadened with a Lorentzian of $0.2 \mathrm{eV}$ and a

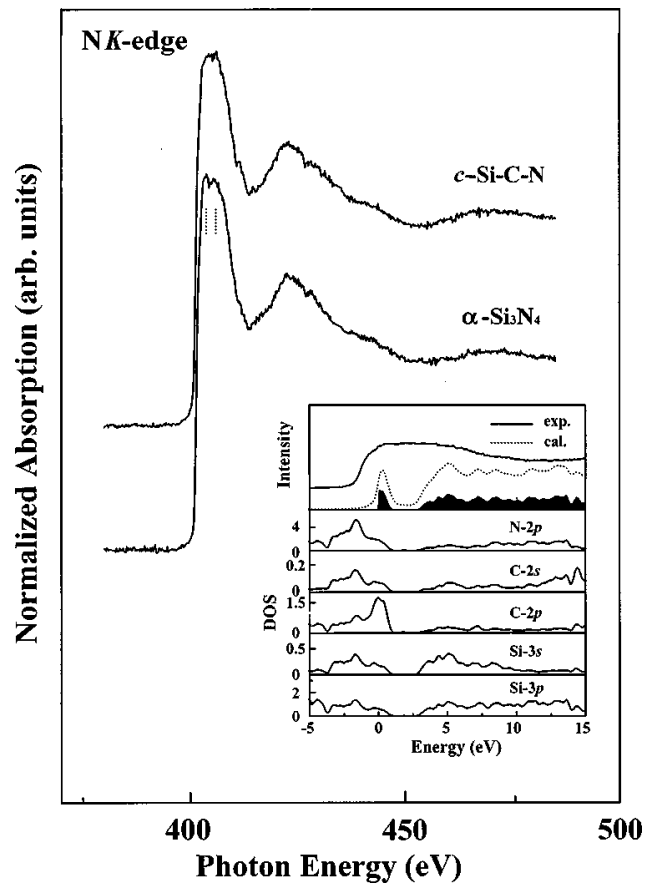

FIG. 3. Normalized fluorescence yield $\mathrm{N} K$-edge absorption spectra of $c$-Si-C-N and $\alpha-\mathrm{Si}_{3} \mathrm{~N}_{4}$. The inset is a comparison of the $\mathrm{N} K$-edge white line features with the calculated nitrogen $p$ projected DOS of $c$-Si-C-N. The darkened area is the theoretical nitrogen $p$-projected DOS above the Fermi level. The upper solid curve is the $\mathrm{N} K$-edge absorption spectra at and above the threshold, and the dashed curve in the middle is the theoretical nitrogen $p$ projected DOS broadened with a Lorentzian and convoluted with a Gaussian function.

Gaussian function of $0.2 \mathrm{eV}$. From this figure, the white line in the $\mathrm{N} K$-edge spectrum of $c$-Si-C-N can generally be assigned to the antibonding $\mathrm{N} 2 p-\mathrm{Si} 3 s p$ and $\mathrm{N} 2 p-\mathrm{C} 2 s p$ hybridized states. The $\mathrm{C} 2 p$ and Si $3 p$ states also have a larger DOS above the threshold region than that of $\mathrm{C} 2 s$ and $\mathrm{Si} 3 s$. Both $c$-Si-C-N and $\alpha-\mathrm{Si}_{3} \mathrm{~N}_{4}$ have nearly identical $\mathrm{N} K$-edge XANES spectra. Since $\alpha-\mathrm{Si}_{3} \mathrm{~N}_{4}$ contains only the $\mathrm{N}-\mathrm{Si}$ bonds, while $c$-Si-C-N contains $\mathrm{N}-\mathrm{Si}$ and $\mathrm{C}-\mathrm{Si}$ bonds, the similarity in the spectra of $c$-Si-C-N and $\alpha-\mathrm{Si}_{3} \mathrm{~N}_{4}$ suggests that the substitution of $\mathrm{N}$ by $\mathrm{C}$ does not alter significantly the local bonding environment of $\mathrm{N}$ atoms. The $\mathrm{N} K$-edge XANES spectrum of $c$-Si-C-N display broad features above the threshold region. However, our theoretical calculations show a sharper feature near threshold, whose intensity drops down quickly at $\sim 1 \mathrm{eV}$, a gap of about $2 \mathrm{eV}$, and then a broad feature that emerges at $\sim 3 \mathrm{eV}$ above the Fermi level. This discrepancy may be due to the fact that $c$-Si-C-N contains not only a majority of the $\alpha-\mathrm{Si}_{3} \mathrm{~N}_{4}$ structure but also some other minor local structures around the absorbing nitrogen atoms, which smeared out the shape white line features. The effect of the deep core hole may also affect the final-state correction, which concerns the calculation of a charged system and is beyond our capability. We believe that nitrogen atoms have similar local environment in both $c$-SiC-N and $\alpha-\mathrm{Si}_{3} \mathrm{~N}_{4}$ but the multiple-local-structure and disorder effects in $c$-Si-C-N cause the broadening of the white line feature in its $\mathrm{N} K$-edge XANES spectra. Please note that the theoretical calculations are concerned with only the perfectly hexagonal structure.

Figure 4 displays the photon-flux-normalized Si $K$-edge 


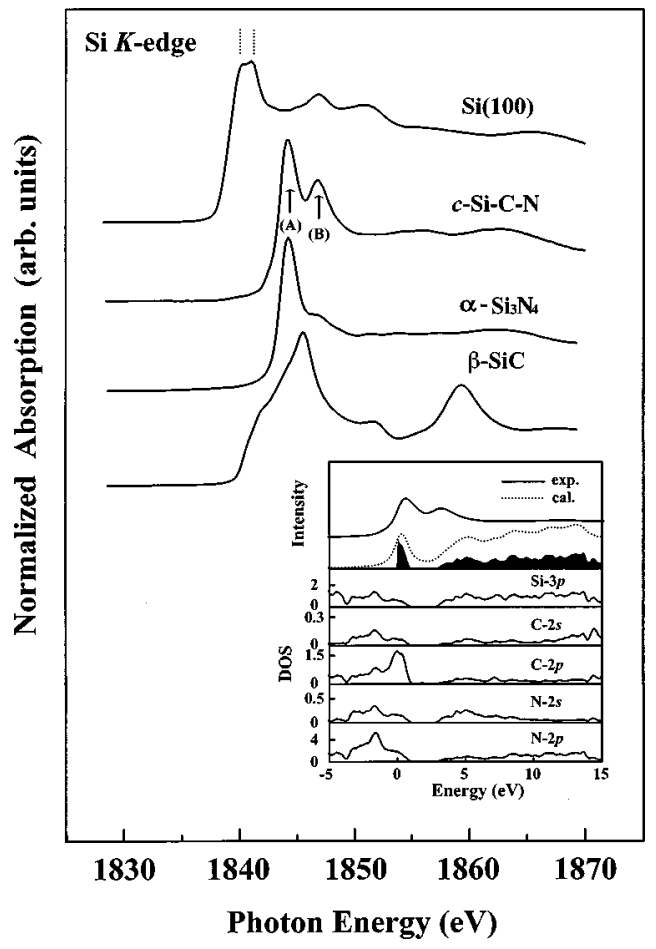

FIG. 4. Normalized Si $K$-edge XANES spectra of $c$-Si(100), $c$-Si-C-N, $\alpha-\mathrm{Si}_{3} \mathrm{~N}_{4}$, and $\beta$-SiC. The inset compares $\mathrm{Si} K$-edge XANES spectra with calculated silicon $p$-projected DOS of $c$-SiC-N. The darkened area shows the theoretical silicon $p$-projected DOS above the Fermi level. The upper solid curve is the Si $K$-edge absorption spectra at and above the threshold, and the dashed curve in the middle is the theoretical silicon $p$-projected DOS broadened with a Lorentzian and convoluted with a Gaussian function.

XANES spectra of $c-\mathrm{Si}(100), c-\mathrm{Si}-\mathrm{C}-\mathrm{N}, \alpha-\mathrm{Si}_{3} \mathrm{~N}_{4}$, and $\beta$-SiC. In the case of $c-\mathrm{Si}(100)$, the $\mathrm{Si} K$-edge XANES spectrum reflects the transition from the $\mathrm{Si} 1 s$ core level to the unoccupied Si $3 p$-derived states. This figure also displays characteristic double-peak features (labeled as two vertical dashed lines) above the edge with an energy separation of about 1.0 $\mathrm{eV}$. Figure 4 also clearly demonstrates that the threshold in the $\mathrm{Si} K$-edge XANES of $c$-Si-C-N shifts toward higher binding energies. In addition, the general line shape of the spectra differs distinctly from that of $c-\operatorname{Si}(100)$. These indicate that the chemical states of the absorbing silicon atoms in $c$-Si-C-N are obviously different than that of $c$-Si(100). Figure 4 also indicates that the $\mathrm{Si} K$-edge XANES spectra of $c$-Si-C-N and $\alpha-\mathrm{Si}_{3} \mathrm{~N}_{4}$ consist of a main peak (labeled A) and a shoulder (labeled B). However, in the $c$-Si-C-N spectrum peak $\mathrm{B}$ is more prominent and peaks $\mathrm{A}$ and $\mathrm{B}$ appear to be well resolved. The prominent peak B in $c$-Si-C-N may be due to the existence of local $\mathrm{Si}-\mathrm{C}$ bonds around silicon atoms because peak $\mathrm{B}$ resembles that of the maximum peak of $\beta$ $\mathrm{SiC}$. This is supported by the results of the $\mathrm{C} K$-edge XANES, which show the occurrence of local C-Si bonds in $c$-Si-C-N. The inset of Fig. 4 presents the Si $K$-edge XANES spectra and calculated unoccupied silicon $p$-projected DOS of $c$-Si-C-N. The darkened area denotes the theoretical silicon $p$-projected DOS above the Fermi level. The upper solid curve represents the $\mathrm{Si} K$-edge absorption spectra, and the dashed curve in the middle is the theoretical silicon $p$ projected DOS broadened with a Lorentzian of $0.48 \mathrm{eV}$ (Ref. 30) and a Gaussian parameter of $0.7 \mathrm{eV}$. Apparently, peaks
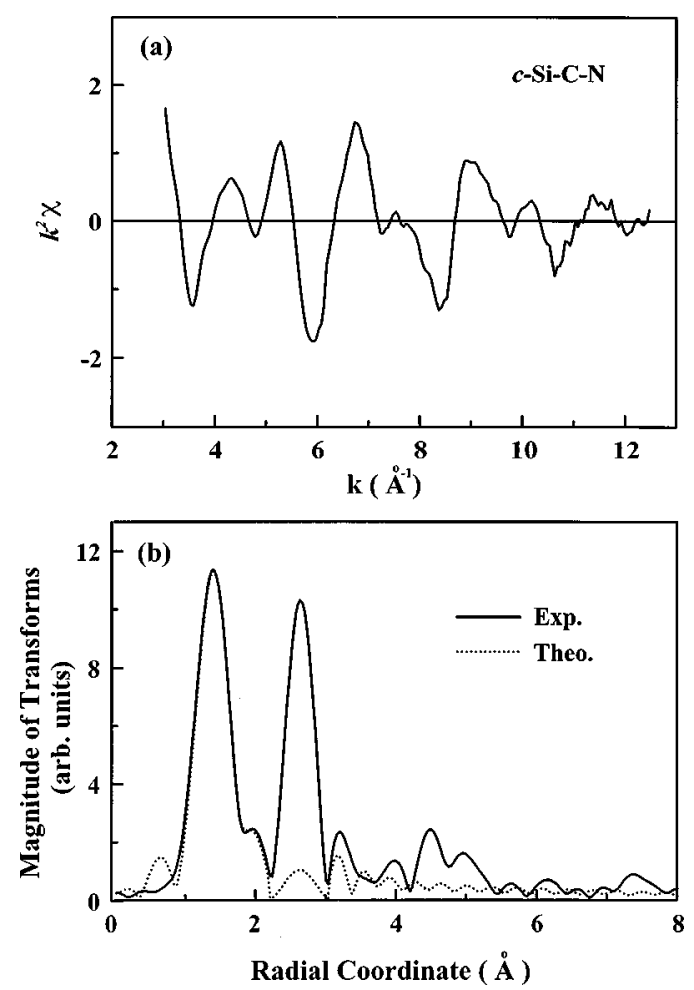

FIG. 5. (a) Normalized $\mathrm{Si} K$-edge EXAFS oscillations $\chi(k)$ weighted by $k^{2}$ of $c$-Si-C-N at room temperature. (b) Magnitude of the Fourier transform of the EXAFS $k^{3} \chi$ data from $k=3.5$ to 12.0 $\AA^{-1}$ (solid line). Final fit of theory to the nearest-neighbor (NN) $\mathrm{Si}-\mathrm{N}$ and Si-C bond lengths (dashed line). Structural parameters of the two NN's obtained by fitting the Si $K$-edge EXAFS spectrum: $N_{\mathrm{Si}-\mathrm{N}}=3 \pm 0.1, R_{\mathrm{Si}-\mathrm{N}}=1.73 \pm 0.01 \AA, \sigma^{2}{ }_{\mathrm{Si}-\mathrm{N}}=(3.8 \pm 0.5) \times 10^{-3} \AA^{2}$. $N_{\text {Si-C }}=1 \pm 0.1, R_{\text {Si-C }}=1.88 \pm 0.01 \AA, \quad \sigma_{\text {Si-C }}^{2}=(3.6 \pm 0.5) \times 10^{-3} \AA^{2}$. $N, R$, and $\sigma^{2}$ are the coordination number, NN distance, and meansquare vibrational amplitude, respectively.

$\mathrm{A}$ and $\mathrm{B}$ in the Si $K$-edge XANES spectrum of $c$-Si-C-N can be attributed to the antibonding Si $3 p-\mathrm{N} 2 s p$ and $\mathrm{Si} 3 p-\mathrm{C} 2 s p$ hybridized states. According to calculations, the $\mathrm{Si} 3 p-\mathrm{N} 2 p$ and $\mathrm{Si} 3 p-\mathrm{C} 2 p$ hybridized states contribute more significantly to the Si $K$-edge XANES spectra. These results agree with our experimental data and are consistent with results of $\mathrm{C}$ and $\mathrm{N} K$-edge XANES, thereby confirming the occurrence of local Si-N and Si-C bonds in $c$-Si-C-N.

This study also attempts to precisely determine the local environment around silicon atoms and to verify the conclusions derived from the $\mathrm{C}, \mathrm{N}$, and $\mathrm{Si} K$-edge XANES spectra and our theoretical calculations of $c$-Si-C-N. The Si $K$-edge EXAFS spectra were measured from $c$-Si-C-N using the sample drain current mode at room temperature. ${ }^{31}$ Figures 5(a) and 5(b) present the normalized EXAFS oscillation $\chi(k)$ weighted by $k^{2}$ for the $\mathrm{Si} K$ edge and the corresponding Fourier transforms (FT's) of the $k^{3} \chi$ data, respectively. Further quantitative analysis performed herein involves combining the multiple-scattering EXAFS computer program FEFF6 (Ref. 32) with the nonlinear least squares fitting computer program FEFFIT. ${ }^{33}$ A fit procedure based on a two-shell model of $\mathrm{Si}-\mathrm{N}$ and $\mathrm{Si}-\mathrm{C}$ pairs is also applied to the first main peak of the FT curves in $c$-Si-C-N. As shown in Fig. 5(b), the quality of the fit for the first main FT peak is quite good. According to this analysis, the $\mathrm{Si}$ atoms are bounded with three nearest-neighbor (NN) Si-N and Si-C bonds. Thus, the 


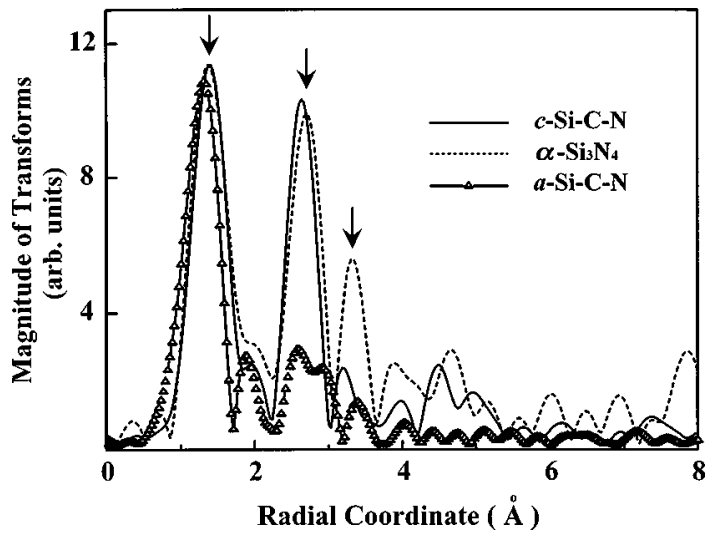

FIG. 6. Magnitude of the Fourier transform of the EXAFS $k^{3} \chi$ data of the Si $K$ edge of $c$-Si-C-N (solid line), $\alpha-\mathrm{Si}_{3} \mathrm{~N}_{4}$ (dashed line), and $a$-Si-C-N (solid line with open triangles) at room temperature.

local atomic structure of $c$-Si-C-N, on average, closely resembles that of the tetrahedral $\mathrm{C}-\mathrm{Si}-\mathrm{N}_{3}$ arrangement. The second main peak centered near $2.7 \AA$ in the FT curves in Fig. 5(b) show the long-ranged ordered atomic arrangement with multiple next-nearest-neighbor $(\mathrm{NNN})$ bonds around silicon atoms in $c$-Si-C-N. Notably, the fitting results for the NN Si-N and Si-C bond lengths in $c-\mathrm{Si}-\mathrm{C}-\mathrm{N}$ were found to be consistent with those presented in Refs. 4 and 5. The general FT spectra of $c$-Si-C-N in Fig. 5(b) closely resemble not only that of Si-C-N nanometric powders reported in Fig. 9 of Ref. 4, but also that of $\alpha-\mathrm{Si}_{3} \mathrm{~N}_{4}$. Figure 6 displays the FT curves obtained from $c$-Si-C-N and $\alpha-\mathrm{Si}_{3} \mathrm{~N}_{4}$. The first main peaks of the FT curves in Fig. 6 (labeled by the first vertical arrow), which corresponds to the NN bond lengths, are nearly at the same position for both $c$-Si-C-N and $\alpha-\mathrm{Si}_{3} \mathrm{~N}_{4}$. But the position of the second FT peak (labeled by the second vertical arrow) for $c$-Si-C-N is shifted slightly to the lower $R$ side relative to that for $\alpha-\mathrm{Si}_{3} \mathrm{~N}_{4}$ though they are still fairly close. The third peak of the FT curves (labeled by the third vertical arrow) for $c$-Si-C-N is also shifted slightly to lower $R$ side and the amplitude is obviously much smaller for $c$-Si-C-N than for $\alpha-\mathrm{Si}_{3} \mathrm{~N}_{4}$. This property can be attributed primarily to the decrease of the structural order and/or the coordination numbers. We would like to mention that the NN Si-N and Si-C bond lengths in $c$-Si-C-N are essentially identical to that in $\alpha-\mathrm{Si}_{3} \mathrm{~N}_{4}$ and $\beta$-SiC, ${ }^{4}$ respectively, which can alternatively be interpreted as an evidence of a mixture of $\alpha-\mathrm{Si}_{3} \mathrm{~N}_{4}$ and $\beta$-SiC phases in the $c$-Si-C-N. However, (1) the XRD data mentioned earlier showed that there was no significant contribution from the $\beta$-SiC phase in the $c$-Si$\mathrm{C}-\mathrm{N}$. (2) The C $K$-edge XANES is suggestive of an average $\mathrm{C}$ environment similar to (but not identical to) that in $\beta$-SiC. It appears (especially from the $\pi^{*}$-like resonance) that not all of the $\mathrm{C}$ could be in the $\beta$-SiC phase. (3) The Si $K$-edge XANES data indicate that most of the $\mathrm{Si}$ are not in $\beta$-SiC phase; in particular the measured XANES spectra of $c$-SiC-N are significantly different than the simple sum of the two distinct Si $K$-edge XANES spectra (in different proportions) of $\alpha-\mathrm{Si}_{3} \mathrm{~N}_{4}$ and $\beta$-SiC. (4) The positions and amplitudes of the first two main peaks in Fig. 6 are quite similar for both $c$-Si-C-N and $\alpha-\mathrm{Si}_{3} \mathrm{~N}_{4}$. These facts support the argument that $c$-Si-C-N has a $\alpha-\mathrm{Si}_{3} \mathrm{~N}_{4}$ structure with a local tetrahedral C-Si- $\mathrm{N}_{3}$ arrangement and is consistent with our theoretical calculations for the $c$-Si-C-N.
As mentioned earlier, there has been a dispute over the atomic structure of the Si-C-N compound. Gheorghiu et al. ${ }^{4}$ suggested the existence of a local C-Si-N $\mathrm{N}_{3}$ arrangement around $\mathrm{Si}$ atoms and it is likely to have the long-range ordered $\alpha-\mathrm{Si}_{3} \mathrm{~N}_{4}$ structure. This is in contrary to that $\mathrm{Si}$ atoms are randomly linked together through the $\mathrm{C}-\mathrm{N}$ network without a long-range order proposed by Ténégal et al..$^{5} \mathrm{In}$ a separate study of the $\mathrm{Si} K$-edge EXAFS for the amorphous $(a)$ Si-C-N film, ${ }^{31}$ it was found that the first peak in the general FT curves for $a$-Si-C-N (also see Fig. 6) shifts slightly toward the lower $R$. Due to larger Debye-Waller factors, the magnitude of the second main peak in the FT curves for $a$-Si-C-N will reduce more significantly than for $c$-Si-C-N and $\alpha-\mathrm{Si}_{3} \mathrm{~N}_{4}$. In fact, the general FT curves for $a-\mathrm{Si}-\mathrm{C}-\mathrm{N}$ is quite similar to that for nanometric Si-C-N powders presented by Ténégal et al. (see Fig. 3 of Ref. 5), which also lacks a long-range order for $R$ values exceeding $\sim 2 \AA$. Therefore, this dispute regarding the local atomic structure (as to whether the Si-C-N system is crystallinelike arrangements or random networks) might be due to the differences in the raw materials and/or the procedures used to prepare the samples. In particular, the local environment around silicon atoms strongly associated with the N/C concentration ratio. ${ }^{7}$ Finally, by combining the XRD data, $\mathrm{C}, \mathrm{N}$, and $\mathrm{Si}$ $K$-edge XANES and Si $K$-edge EXAFS results and theoretical calculations for $c$-Si-C-N, we can conclude that the silicon atoms in $c$-Si-C-N preferentially have a similar local structure as in $\alpha-\mathrm{Si}_{3} \mathrm{~N}_{4}$. This confirms the existence of a local tetrahedral C-Si- $\mathrm{N}_{3}$ arrangement and a long-range ordered atomic structure rather than that silicon atoms randomly linked together through the C-N network without long-range order.

\section{CONCLUSION}

Results obtained from $\mathrm{C} K$-edge XANES spectra and first-principles calculations demonstrate that the broad feature in the XANES energy range can be assigned to the antibonding $\mathrm{C} 2 p-\mathrm{Si} 3 s p$ hybridized states and the $\mathrm{C} 2 p-\mathrm{N} 2 s p$ hybridized states. The resonance peak, which can be described by the Frenkel-type exciton model, can be attributed to the core exciton of carbon atoms in $c-\mathrm{Si}-\mathrm{C}-\mathrm{N}$. The nearly identical features in the $\mathrm{N} K$-edge XANES spectra for both $c$-Si-C-N and $\alpha-\mathrm{Si}_{3} \mathrm{~N}_{4}$ show that both $c$-Si-C-N and $\alpha-\mathrm{Si}_{3} \mathrm{~N}_{4}$ have similar local environments around the nitrogen atoms. The Si $K$-edge XANES and EXAFS spectra confirm the existence of a local tetrahedral C-Si- $\mathrm{N}_{3}$ arrangement with a long-range ordered crystallinelike structure around silicon atoms in $c$-Si-C-N. We note that the above explanations of the local electronic and atomic structures are based on polycrystalline $c$-Si-C-N, which mainly has a homogeneous hexagonal structure with corner-linked tetrahedra.

\section{ACKNOWLEDGMENTS}

One of the authors (W.F.P.) would like to thank the National Science Council of the R.O.C. for financially supporting this research under Contract No. NSC 87-2613-M-032003. SRRC is also appreciated for allowing us to use their HSGM and DCM beamlines to perform this study. 
*Author to whom all correspondence should be addressed.

${ }^{1}$ M. Dayan, J. Vac. Sci. Technol. A 3, 361 (1985); E. C. Paloura, J. Lagowski, and H. C. Gatos, J. Appl. Phys. 69, 3995 (1991).

${ }^{2}$ M. L. Cohen, Phys. Rev. B 32, 7988 (1985); A. Y. Liu and M. L. Cohen, Science 245, 841 (1989).

${ }^{3}$ Z. He, G. Carter, and J. S. Colligon, Thin Solid Films 283, 90 (1996).

${ }^{4}$ A. Gheorghiu, C. Sénémaud, H. Roulet, G. Dufour, T. Moreno, S. Bodeur, C. Reynaud, M. Cauchetier, and M. Luce, J. Appl. Phys. 71, 4118 (1992).

${ }^{5}$ F. Ténégal, A. M. Flank, and N. Herlin, Phys. Rev. B 54, 12029 (1996).

${ }^{6}$ A. Bendeddouche, R. Berjoan, E. Bêche, T. Merle-Mejean, S. Schamm, V. Seriin, G. Taillades, A. Pradel, and R. Hillel, J. Appl. Phys. 81, 6147 (1997).

${ }^{7}$ M. Driss-Khodja, A. Gheorghiu, G. Dufour, H. Roulet, C. Sénémaud, and M. Cauchetier, Phys. Rev. B 53, 4287 (1996).

${ }^{8}$ J. F. Morar, F. J. Himpsel, G. Hollinger, G. Hughes, and J. L. Jordan, Phys. Rev. Lett. 54, 1960 (1985).

${ }^{9}$ W. F. Pong, Y. K. Chang, R. A. Mayanovic, G. H. Ho, H. J. Lin, S. H. Ko, P. K. Tseng, C. T. Chen, A. Hiraya, and M. Watanabe, Phys. Rev. B 53, 16510 (1996).

${ }^{10}$ L. C. Chen, C. Y. Yang, D. M. Bhusari, K. H. Chen, M. C. Lin, J. C. Lin, and T. J. Chuang, Diamond Relat. Mater. 5, 514 (1996).

${ }^{11}$ L. L. Snead, M. Osborne, and K. L. More, J. Mater. Res. 10, 736 (1995); L. Hozer and Y. M. Chiang, ibid. 11, 2346 (1996).

${ }^{12}$ M. B. Kruger, J. H. Nguyen, Y. M. Li, W. A. Caldwell, M. H. Manghnani, and R. Jeanloz, Phys. Rev. B 55, 3456 (1997).

${ }^{13}$ R. V. Kasowski, M.-H. Tsai, T. N. Rhodin, and D. D. Chambliss, Phys. Rev. B 34, 2656 (1986); M.-H. Tsai and K. C. Hass, ibid. 51, 14616 (1995); K. C. Hass, M.-H. Tsai, and R. V. Kasowski, ibid. 53, 44 (1996).

${ }^{14}$ S. L. Cunningham, Phys. Rev. B 10, 4988 (1974).

${ }^{15}$ International Tables for X-ray Crystallography, edited by N. F. M. Henrand K. Lonsdal (Kynoch, Birmingham, 1969), Vol. 1.

${ }^{16}$ Table of Periodic Properties of the Elements (Sargent-Welch Sci- entific Company, Skokie, IL, 1980).

${ }^{17}$ Y. Ma, N. Wassdahl, P. Skytt, J. Guo, J. Nordgren, P. D. Johnson, J. E. Rubensson, T. Boske, W. Eberhardt, and S. D. Kevan, Phys. Rev. Lett. 69, 2598 (1992).

${ }^{18}$ J. Nithianandam, J. C. Rife, and H. Windischmann, Appl. Phys. Lett. 60, 135 (1992).

${ }^{19}$ A. Gutierrez, M. F. Lopez, I. Garcia, and A. Vazquez, J. Vac. Sci. Technol. A 15, 294 (1997).

${ }^{20}$ J. Robertson, Philos. Mag. B 66, 615 (1992).

${ }^{21}$ V. S. Veerasamy, J. Yuan, G. A. J. Amaratunga, W. I. Milne, K. W. R. Gilkes, W. Weiler, and L. M. Brown, Phys. Rev. B 48, 17954 (1993).

${ }^{22}$ F. Weich, J. Widany, and Th. Frauenheim, Phys. Rev. Lett. 78, 3326 (1997).

${ }^{23}$ K. A. Jackson and M. R. Pederson, Phys. Rev. Lett. 67, 2521 (1991).

${ }^{24}$ Y. Ma, P. Skytt, N. Wassdahl, P. Glans, D. C. Mancini, J. Guo, and J. Nordgren, Phys. Rev. Lett. 71, 3725 (1993).

${ }^{25}$ J. Stöhr, NEXAFS Spectroscopy (Springer-Verlag, Berlin, 1992).

${ }^{26}$ M. Jaouen, G. Tourillon, J. Delafond, N. Junqua, and G. Hug, Diamond Relat. Mater. 4, 200 (1995).

${ }^{27}$ D. Y. Lin, C. F. Li, Y. S. Huang, Y. C. Jong, Y. F. Chen, L. C. Chen, C. K. Chen, K. H. Chen, and D. M. Bhusari, Phys. Rev. B 56, 6498 (1997).

${ }^{28}$ S. T. Pantelides and F. C. Brown, Phys. Rev. Lett. 33, 298 (1974); S. L. Hulbert, B. A. Bunker, F. C. Brown, and P. Pianetta, Phys. Rev. B 30, 2120 (1984).

${ }^{29}$ J. Robertson, Philos. Mag. B 63, 47 (1991).

${ }^{30}$ See, Unoccupied Electronic States, edited by J. C. Fuggle and J. E. Inglesfield (Springer-Verlag, Berlin, 1992), Appendix B.

${ }^{31}$ W. F. Pong, Y. K. Chang, H. H. Hsieh, and T. E. Dann (unpublished).

${ }^{32}$ J. J. Rehr, J. M. deLeon, S. I. Zabinsky, and R. C. Albers, J. Am. Chem. Soc. 113, 5135 (1991); J. J. Rehr, S. I. Zabinsky, and R. C. Albers, Phys. Rev. Lett. 69, 3397 (1992).

${ }^{33}$ A. I. Frenkel, E. A. Stern, M. Qian, and M. Newville, Phys. Rev. B 48, 12449 (1993). 